\title{
CUIDADO DE LA SALUD DEL ADULTO MAYOR EN UNA COMUNIDAD SEMIRURAL DESDE UNA METODOLÓGICA DE PARTICIPACIÓN ACCIÓN INVESTIGACIÓN CENTRADA EN LA OCUPACIÓN
}

HEALTH CARE OF THE ELDERLY IN A SEMI-RURAL COMMUNITY WITH A METHODOLOGY OF PARTICIPATORY-ACTION-RESEARCH FOCUSED ON THE OCCUPATIONAL PERSPECTIVE

\section{Cristina Bolaños', Gabriel Martinez ${ }^{2}$ y Gregorio Ramos ${ }^{3}$}

\section{Resumen}

El objetivo del estudio fue identificar las necesidades del cuidado de la salud de los adultos mayores en una comunidad semi - rural, las redes de apoyo existentes y desarrollar un proyecto de cuidado de salud dirigido a esta población desde un enfoque ocupacional. Se siguió una metodología mixta de tres fases: En la Fase I se utilizó un diseño descriptivo, transversal y correlacional. En la Fase Il se utilizó un diseño mixto cuantitativo y cualitativo y en la Fase III una metodología cualitativa. En las Fases II y III se utilizó una metodología de Investigación-Acción-Participación y Rehabilitación Basada en la Comunidad.

Resultados.

Se identificaron los recursos y necesidades ocupacionales y de salud de los adultos mayores residentes en la comunidad. Estos recursos y necesidades sirvieron de base para realizar un programa de sensibilización, capacitación y atención rehabilitatoria a adultos mayores, implementado con la participación de cuidadores primarios. Se identificaron los recursos y necesidades comunitarias en cuanto a sus redes de apoyo, esto generó la creación de un grupo de apoyo para los adultos mayores en situación de vulnerabilidad.

Esta experiencia permitió vincular a diferentes actores en el cuidado de la salud del adulto mayor, creando conciencia del valor de su propio conocimiento y su aportación al entretejido social de la comunidad. El involucramiento de los pasantes de licenciatura y maestría de terapia ocupacional a lo largo del proyecto, permitió ampliar el número de beneficiarios, capacitar en rehabilitación basada en la comunidad a cuidadores primarios, además de darle a los estudiantes la oportunidad de involucrarse y colaborar directamente en la comunidad, identificando las necesidades ocupacionales de la población para intervenir en forma más efectiva.

\section{Palabras Claves}

Adultos mayores, Redes sociales, Participación Social, Terapia Ocupacional.

Doctora en Investigación Psicológica, Maestría en Desarrollo Humano, Lic. En Terapia Ocupacional. Coordinadora Maestría en Terapia Ocupacional, Instituto de Terapia Ocupacional. Av. San Antonio 341-1, Col. San Pedro de los Pinos. Tel: 525555986254 . Email: cbolanos@ ito-edu.org.mx.

Maestro en Terapia Ocupacional, Licenciado en Fisioterapia. Instituto de Terapia Ocupacional. Email: gbrlmartinez149@gmail.com. Asesor estadístico. Instituto de Terapia Ocupacional. Email: ramosgregorio57@gmail.com. 


\section{Abstract}

The objective of the study was to identify the health care needs of the elderly in a semi - rural community, their existing support networks and to develop a healthcare project for this population from an occupational perspective.

A mixed, three-phase methodology was used. In Phase I, a descriptive, transverse and correlational design was employed, followed by a combined quantitative and qualitative design in Phase II and a qualitative methodology in Phase III. In the latter two phases, a Participatory-Action-Research Methodology and Community Based Rehabilitation was used.

Results.

The occupational resources and health needs of the elderly in the community were identified. These resources and needs became the basis for the development of an awareness and training program for the caregivers. A rehabilitation support program was also implemented. Social network resources and needs were identified and generated a support group for the elderly in a vulnerable situation.

This project allowed to connect different actors involved in the healthcare of the elderly. It also raised awareness among the elderly regarding the value of their own knowledge and their contribution to the social fabric of the community.

The involvement of undergraduate and graduate interns in occupational therapy throughout the project facilitated covering different types of health needs as well as to expand the number of beneficiaries. It also gave students the opportunity to get involved and to work directly in the community through the identification of the occupational needs of the population as a means to intervene more effectively.

\section{Key Words:}

Elderly, Social Networks, Social participation, Occupational Therapy

Recepcionado: 22/11/2016

Aceptado: 31/05/2017 


\section{INTRODUCCIÓN}

De acuerdo con estadísticas del Consejo Nacional de Población (CONAPO, 2015) el número de adultos mayores mexicanos se duplicó en las últimas décadas, pasando de 5 a 11.7 millones de personas de 60 años y más. Por su parte, la Comisión Económica para la América Latina (CEPAL, 2015) estima que para 2030 uno de cada cinco habitantes de la capital del país será una persona mayor de 60 años.

Los adultos mayores son un grupo vulnerable por el aumento de la discapacidad por envejecimiento, por las enfermedades crónicas degenerativas que les aquejan, y por su creciente dependencia de terceros. De acuerdo con la Encuesta Nacional de Salud (ENSANUT, 2012) el total de adultos mayores con discapacidad es de $47.8 \%$; de los cuales entre los 60-69 años, el 36\% presenta algún tipo de discapacidad y en los mayores de 80 años, esta aumenta a un $77 \%$. Las principales limitaciones encontradas en dicha encuesta fueron funcionales $22 \%$, cognitivas $18 \%$ y visuales $16 \%$.

En un diagnóstico realizado por la Secretaría de Desarrollo Social (SEDESOL) y el Instituto Nacional de Estadística y Geografía Informática (INEGI 2010) se encontró que en los adultos mayores de 60 años y más, las causas principales de vulnerabilidad son la insuficiencia de ingresos, la falta de protección social, el deterioro y la disminución de sus activos, el aceleramiento del deterioro natural, la baja calidad de vida, la exclusión social y una mayor dependencia de terceros. En la Encuesta Nacional de Empleo y Seguridad Social (ENESS) (INEGI, 2013), se menciona que sólo $26 \%$ de los adultos mayores se encuentran pensionados.

\section{Atención del Adulto Mayor desde la Perspectiva Ocupacional}

La práctica comunitaria de la terapia ocupacional refleja la importancia que tiene la ocupación en la formación de comunidades sanas y su inclusión en actividades de la vida cotidiana de las personas (Doll, 2010). Conocer la relación entre la ocupación y los problemas de salud, permite establecer las prioridades y necesidades de salud de las personas que la habitan. Fazzio (2008) menciona que existe un sistema dinámico entre comunidad y el ambiente que afecta positiva o negativamente las ocupaciones y con ella la salud de la comunidad. Las comunidades son los escenarios donde las personas residen, construyen relaciones y participan en prácticas de salud
(Scaffa, 2001). La práctica de la terapia ocupacional en comunidades requiere de programas únicos para cada comunidad y del trabajo de los terapeutas ocupacionales dentro de la comunidad. La práctica comunitaria explora el rol de la ocupación como formador de la sociedad y la vida diaria (Filder 2001, p.7) y las propuestas de la terapia ocupacional van enfocadas a las necesidades de la población (Mc Coll, 1998).

Los programas de terapia ocupacional basados en comunidades han atendido de manera eficaz problemáticas de salud y de calidad de vida de los adultos mayores. El programa Rediseño de las Ocupaciones (Clark et al., 1997) demostró que hubo diferencias significativas entre los grupos de adultos mayores que vivían en la comunidad y participaron en el programa orientado a mantener la salud a través del rediseño de sus ocupaciones y de aquellos que sólo participaron en programas recreativos y de manualidades y que siguieron las rutinas que habían Ilevado hasta el momento. (Matuska, 2003). Graff (2006 y 2008) evaluó la eficacia de un programa de terapia ocupacional comunitario para personas con demencia y la sensación de competencia de sus cuidadores. Los resultados fueron significativos, ya que mejoró la funcionalidad del adulto mayor en la vida cotidiana y disminuyó el estrés en los cuidadores. Cummings et al. (1999) determinó la eficacia de visitas domiciliarias de terapia ocupacional para identificar y reducir riesgos de caídas en adultos en comunidades, que habían sido recientemente hospitalizados.

Liddle et al. (1996), determinó el efecto en la calidad de vida e independencia de los adultos mayores, después de recibir una intervención ocupacional para la modificación del hogar y servicios comunitarios. Gitlin (2009) se refiere a las formas de "adaptación ambiental" en el ámbito tecnológico, estructural, material y en la estructura del hogar, mientras que Issac (1990) enfatiza los aspectos actitudinales de los miembros de la familia abordando la necesidad de "trabajar con las familias" en el enfoque comunitario de Terapia Ocupacional.

\section{Problemática del adulto mayor en San Lorenzo Acopilco}

El pueblo de San Lorenzo Acopilco (SLA) se ubica al suroeste de la Delegación Cuajimalpa, en la ciudad de México, en un área montañosa de clima semi - frío y húmedo. Presenta una población aproximada de 23000 habitantes, de los cuales 1510 son adultos mayores de 60 años (INEGI, 2010). La mayoría de los hogares cuentan con servicios públicos básicos, como agua potable, 
drenaje, electricidad y teléfono. Por estar ubicado en la montaña, las calles del poblado presentan subidas bajadas, pocas banquetas, irregularidad del pavimento lo que dificulta el traslado, movilidad y vinculación de sus adultos mayores con su comunidad.

SLA cuenta con un espacio comunitario destinado para el desarrollo y bienestar de los adultos mayores, organizado y apoyado por SEDESOL. Funciona una vez a la semana durante la mañana. Además existe un centro de la propia comunidad en donde se dan clase de manualidades y diferentes tipos de ejercicios, principalmente dirigidos a jóvenes y niños.

\section{MARCO CONCEPTUAL}

Promoción de la salud y perspectiva ocupacional

La promoción de la salud ha sido definida como "e proceso que permite a las personas aumentar el contro sobre su salud y mejorarla" (OMS. 1986, p.2.). Se consideran seis categorías determinantes de la salud: biológica, conductuales, entorno social y físico, políticos y acceso a cuidados de salud. A su vez, la Carta de Otawa (OMS 1986) describe cinco áreas de acción de la promoción de la salud, tales como: creación de políticas públicas de salud, creación de ambientes favorables, reforzamiento de la acción comunitaria, desarrollo de las aptitudes personales y la reorientación de los servicios sanitarios.

Cabe señalar que se considera a la promoción de la salud como un proceso, cuyas etapas no siempre están claramente definidas, Letts y cols (1993) describieron las fases iniciales del proceso, que incluyen lo siguiente: a) formación de redes, b) consulta (para identificar problema de salud), c) colaboración (para seleccionar un problema específico a tratar), d) planificación (acciones y desarrollar objetivos).

\section{Rehabilitación basada en la comunidad}

La rehabilitación basada en la comunidad fue iniciada por la OMS en 1978, con el objetivo de dar acceso a los servicios de rehabilitación para las personas con discapacidad en países de bajos y medianos ingresos con sus medios locales.

Para la práctica de este tipo de rehabilitación, es esencial la participación de la comunidad en el proceso de desarrollo, planeación, puesta en práctica y la supervisión (OMS 2012). La rehabilitación basada en la comunidad tiene marcos comunes dirigidos a salud, educación, subsistencia, participación social y fortalecimiento.

\section{Redes sociales de apoyo para enfrentar los problemas de salud}

El término redes sociales se refiere al entretejido de relaciones sociales en las que los individuos se desenvuelven. Estas redes cumplen con diferentes funciones desde laborales, hasta de apoyo que contribuyen al bienestar del individuo. Las redes sociales de apoyo pueden orientarse a dar respaldo emocional (empatía, cuidado, confianza), instrumental (servicios y ayudas tangibles directamente a la persona que lo necesita), informativo (consejos, sugerencias e información para que la persona enfrente los problemas) o de reconocimiento (FUNBAM 2005), (Glaz y cols 2008).

Las redes sociales de apoyo se clasifican en primarias, constituidas por familias, amigos y vecinos. En secundarias extra familiares, que son aquellas conformadas en el ambiente externo a la familia y en terciarias, que son las institucionales formales. Las primarias satisfacen necesidades de interacción social, tanto entre los miembros de la familia como las que éstos mantienen con vecinos. Las secundarias se generan cuando, las personas de edad no disponen de ayudas o apoyos en la cantidad y calidad requerida para cubrir sus necesidades básicas en sus hogares de origen y/o de adscripción. Éstas suelen formarse a través de grupos locales en organizaciones altruistas, instituciones de beneficencia, o bien con organizaciones civiles que desarrollan algún programa o proyecto asistencial y/o promocional en la comunidad (FUNBAM 2005).

Diferentes estudios muestran que el participar en este tipo de redes tiene como beneficio el recrear un sentido de pertenencia y una noción de colectividad, establecer vínculos de amistad, fortalecer su autoestima reconociendo sus propios recursos y capacidad para aportar (Antonucci \& Jackson, 1990, Heaney, C. \& Israel, 2008) y "el desempeño de roles significativos constituyen un elemento clave en su calidad de vida" (Guzman, et al 2003, p 47).

El objetivo general del estudio fue identificar las necesidades del cuidado de la salud de los adultos mayores y las redes de apoyo existentes y generar aquellas que se identificaran como prioritarias para el cuidado de la salud del adulto mayor en SLA, a través de la participación activa de los miembros de la misma comunidad.

Para esto, se establecieron como objetivos específicos:

A. Identificar indicadores del cuidado de la salud y calidad de vida.

B. Sensibilizar a la comunidad sobre el cuidado de la salud. 
C. Identificar las redes de apoyo existentes para el cuidado de la salud del adulto mayor.

D. Capacitar a cuidadores primarios en el cuidado y mantenimiento de la salud desde la perspectiva ocupacional.

Como resultado de la detección de necesidades se dentificó la necesidad de:

E. Ofrecer apoyo a los adultos mayores enfermos o solos referidos por la propia comunidad para su atención.

Posteriormente, hacia el final de la intervención, conjuntamente con el grupo que había recibido capacitación, se identificó la necesidad de:

F. Iniciar la organización de un grupo autogestivo para el cuidado de la salud del adulto mayor.

\section{MÉTODO}

Este estudio utilizó una metodología mixta de tres fases, como se muestra en el gráfico 1.

Fase I: Diagnóstico situacional.

Fase II: Sensibilización, capacitación y atención a problemas rehabilitatorios que impactan el desempeño ocupacional.

Fase III: Identificación de redes de apoyo para el cuidado a la salud del adulto mayor existentes en la comunidad y tipo de redes que aun se requieren.

\section{FASE I: Diagnóstico situacional}

Objetivo General: Identificar las condiciones de salud de los adultos mayores de SLA, así como aquellas ocupaciones significativas que mantienen o han dejado de realizar y las redes de apoyo con las que cuentan.

Gráfico 1: Metodología.

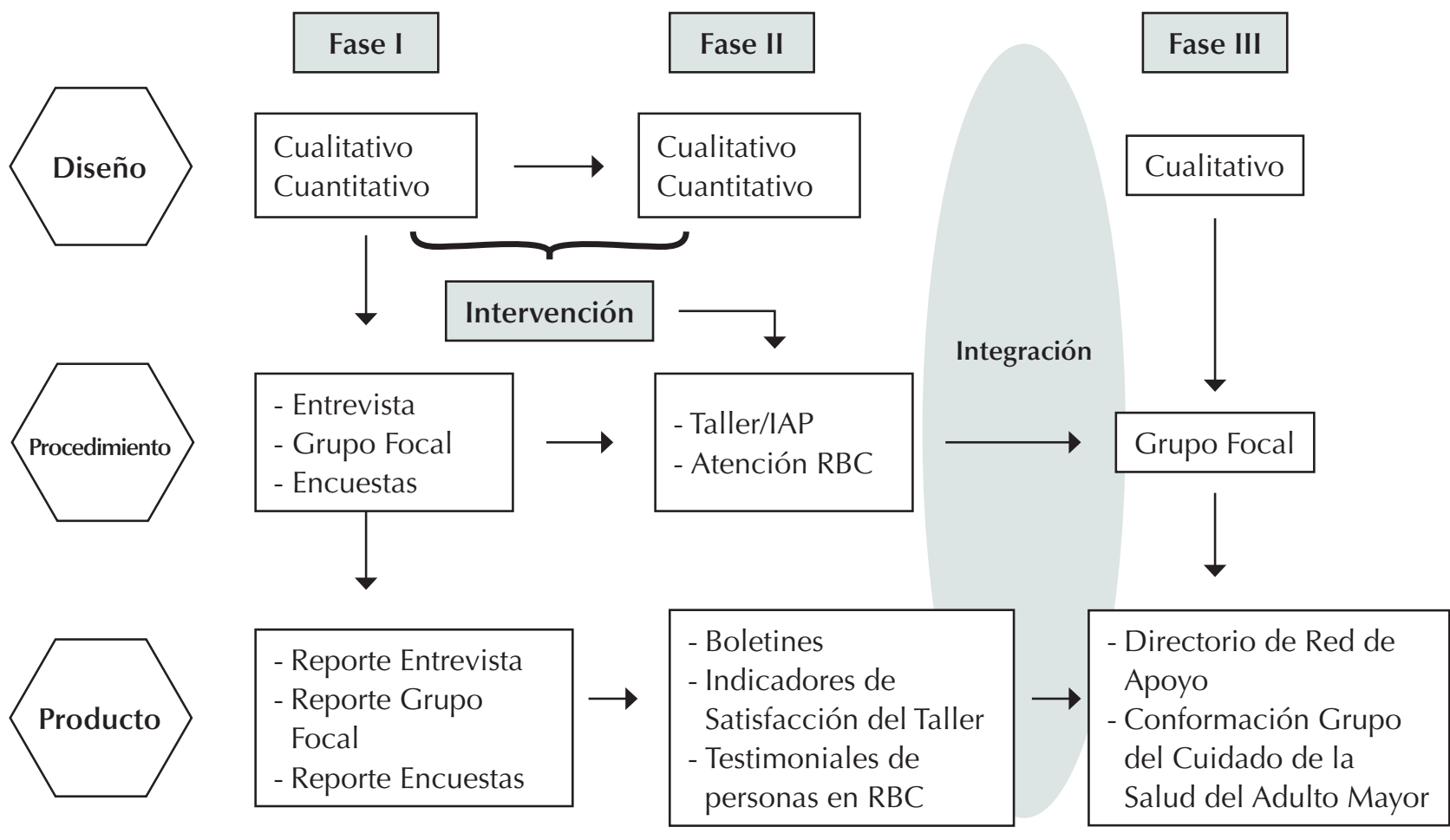

Descripcion conceptual del diseño de estudio 
Instrumentos para la obtención de datos:

- Entrevista al Párroco del pueblo.

- Grupo focal con el grupo parroquial encargado de visitar las casas de los enfermos.

- Encuesta para adultos mayores.

\section{Técnicas de análisis}

En la Fase I se utilizó un diseño mixto de tipo cualitativo para la entrevista con el Párroco para conocer su percepción de las necesidades del adulto mayor en la comunidad y tener acceso a personas claves en ella.

Cuando se le presentó al Párroco el proyecto orientado a crear una red de apoyo al adulto mayor de SLA, externó su preocupación por que consideraba que era un grupo que empezaba a estar relegado en la comunidad, con pocos servicios de salud accesibles, especialmente cuando avanzaba en edad. Se mostró particularmente interesado en dar apoyo al adulto mayor que dejaba de asistir a los servicios parroquiales por problemas de salud y consideraba que ampliar el conocimiento de los Ministros Extraordinarios de la Eucaristía (MECES) ayudaría a ampliar esa red para darle a los adultos mayores mayo presencia de la Parroquia en sus propias casas. Gracias a su apoyo se realizó un grupo focal con el grupo paroquial de los Ministros Extraordinarios de la Eucaristía.

En el grupo focal al que asistieron 17 Ministros, estos mostraron conocer a todos los adultos mayores de la comunidad y externaron su preocupación por quienes se encuentran enfermos, los que no cuentan con redes de apoyo o se encuentran solos enfrentando problemas en sus cuidados básicos. A pesar del interés que manifestaron cuando se les presentó la propuesta de extender los servicios que proporcionaban para crear redes de apoyo para trabajar con los adultos mayores, se percibió rechazo de su parte para involucrarse y apoyar el proyecto principalmente por estar sobrepasados por el número de actividades que realizan para la Parroquia. Esto influyó fuertemente en el proyecto, el cual requirió reorientarse en la forma como se pensaba acercar al adulto mayor.

Debido a ello, se aprovechó que las personas adultas mayores se reunían para recibir el apoyo alimenticio, por lo que tres examinadores levantaron la encuesta para conocer sus necesidades de cuidado de la salud.

A nivel cuantitativo se realizó un estudio descriptivo, transversal y correlacional para conocer la percepción de salud y los apoyos que los adultos mayores consideran que tienen disponibles. Los datos fueron obtenidos de la encuesta.

\section{Resultados}

En la tabla 1 se describen las variables sociodemográficas de los participantes en el diagnóstico situacional. Como se puede ver, el $22 \%$ de los adultos mayores encuestados estaban en el rango de edad de 65 a 74 años $52 \%$ en el de 75 a 84 años; y $26 \%$ en el rango de 85 años en adelante. El $70 \%$ fueron mujeres y $30 \%$ fueron hombres, y sólo el $2 \%$ vive solo.

Tabla 1. Características Sociodemográficas.

\begin{tabular}{|l|l|c|c|}
\hline Categoria & 65 a 74 años & N & $\%$ \\
\hline \multirow{4}{*}{ Edad } & 75 a 84 & 11 & 22 \\
\cline { 2 - 4 } & 85 y más & 26 & 52 \\
\hline \multirow{4}{*}{ Sexo } & Hombre & 13 & 26 \\
\cline { 2 - 4 } & Mujer & 15 & 30 \\
\hline \multirow{4}{*}{ Vocalidad } & Otra & 35 & 70 \\
\cline { 2 - 4 } & La Pila & 1 & 2 \\
\cline { 2 - 4 } & Acopilco & 4 & 8 \\
\hline \multirow{4}{*}{} & Sólo & 45 & 90 \\
\cline { 2 - 4 } & Esposo/a & 31 & 2 \\
\cline { 2 - 4 } & Hijos/familia & 8 & 16 \\
\cline { 2 - 4 } & Otras personas & 10 & 20 \\
\hline
\end{tabular}

Variables sociodemográficas de los adultos mayores que participaron en la encuesta.

Como se observa en la tabla 2, se encontró que e $46 \%$ reportó trastornos en su movilidad y enfermedades musculo esqueleticas, 32\% hipertensión y diabetes. En relación al número de enfermedades por adulto mayor el 50\% reportó sólo una enfermedad, $26 \%$ reportó dos tipos, el 14\% reportó tres simultáneas y sólo el 10\% no reportó tener enfermedades crónicas. No se encontraron diferencias significativas por edad y sexo. 
Tabla 2. Prinicipales enfermedades

\begin{tabular}{|l|c|c|}
\hline Patología & $\mathbf{n}$ & $\%$ \\
\hline Hipertensión & 16 & 32 \\
\hline Diabetes Mellitus & 16 & 32 \\
\hline $\begin{array}{l}\text { Trastornos y enfermedades del sistema } \\
\text { músculo esquelético }\end{array}$ & 23 & 46 \\
\hline Enfermedades cardiacas & 2 & 4 \\
\hline Enfermedades del sistema circulatorio & 2 & 4 \\
\hline Trastornos visuales & 3 & 6 \\
\hline Neoplasias & 2 & 4 \\
\hline Enfermedades intestinales & 1 & 4 \\
\hline Enfermedades hepáticas & 1 & 2 \\
\hline Enfermedades del riñón y de los uréteres & 1 & 2 \\
\hline Neuropatías y poli neuropatías & 1 & 2 \\
\hline $\begin{array}{l}\text { Enfermedades del aparato genital } \\
\text { masculino }\end{array}$ & 1 & 2 \\
\hline
\end{tabular}

Se presenta la relación del tipo de enfermedades que padecen los adultos mayores encuestados.

A continuación se puede observar en la gráfica 2, el $68 \%$ mencionó tener dificultades y problemas visuales, $48 \%$ auditivos y de movilidad, $14 \%$ de memoria y sólo un $4 \%$ táctiles. Al preguntarles qué tanto estos problemas los limitaban para realizar sus actividades, el $46 \%$ mencionó que mucho, $34 \%$ poco y $10 \%$ nada.

Gráfica 2. Dificultades y problemas

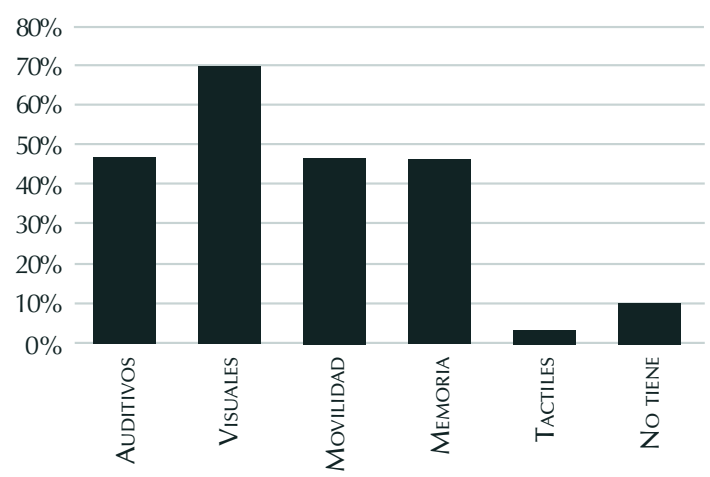

Dificultades y problemas que los adultos mayores consideran que les están afectando para realizar actividades significativas.

En la tabla 3 se presentan las ocupaciones significativas que realizan los adultos mayores en Acopilco.
Tabla 3. Ocupaciones significativas.

\begin{tabular}{|c|c|c|c|}
\hline \multicolumn{2}{|c|}{ Categoría } & $\mathrm{n}$ & $\%$ \\
\hline \multirow{5}{*}{$\begin{array}{ll}0 & 0 \\
\frac{\pi}{0} & \overline{0} \\
\frac{0}{0} & 0 \\
\frac{1}{3} & 0 \\
U & \frac{1}{0} \\
0 & 0\end{array}$} & Caminar & 10 & 20 \\
\hline & Comer & 1 & 2 \\
\hline & Vestirse & 1 & 2 \\
\hline & Bañarse & 1 & 2 \\
\hline & Hacer ejercicio & 5 & 10 \\
\hline \multirow{8}{*}{$\begin{array}{l}\frac{0}{0} \\
\frac{0}{0} \\
: \\
0 \\
0 \\
0 \\
0 \\
0 \\
0 \\
0 \\
0\end{array}$} & Cocinar, preparar alimentos & 20 & 40 \\
\hline & Sembrar o cosechar & 8 & 16 \\
\hline & Limpiar la casa & 14 & 28 \\
\hline & Planchar & 3 & 6 \\
\hline & Lavar ropa & 11 & 22 \\
\hline & Conducir & 3 & 6 \\
\hline & Atender negocio & 6 & 12 \\
\hline & Cuidar a niños & 8 & 16 \\
\hline \multirow{8}{*}{ 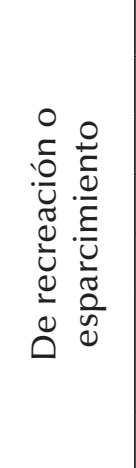 } & Coser, bordar o tejer & 18 & 36 \\
\hline & Alimentar a los animales & 4 & 8 \\
\hline & Ver la televisión & 7 & 14 \\
\hline & Bailar & 1 & 2 \\
\hline & Escuchar música & 4 & 8 \\
\hline & Rezar & 1 & 2 \\
\hline & Leer & 4 & 8 \\
\hline & Ir a misa & 12 & 24 \\
\hline \multirow{4}{*}{ 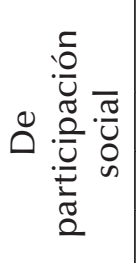 } & $\begin{array}{l}\text { Platicar, convivir con familiares } \\
\text { o amigos }\end{array}$ & 5 & 10 \\
\hline & Ir a fiestas & 2 & 4 \\
\hline & Salir de paseo & 11 & 22 \\
\hline & Visitar a familiares o amigos & 7 & 14 \\
\hline
\end{tabular}

Categorización de las ocupaciones de los adultos encuestados

Sólo se encontraron diferencias estadísticamente significativas en relación a género, en la actividad productiva de cocinar, en donde la diferencia entre hombre y mujeres tuvo una significancia de $p<0.004$. No se obtuvieron diferencias significativas en cuanto a edad en las diferentes áreas ocupacionales realizadas. 


\section{Red de apoyo primaria}

Con respecto a si los adultos mayores requieren ayuda para preparar sus alimentos, $62 \%$ dijo no necesitar ayuda y el $38 \%$ restante dijo contar con ella para realizar esta actividad. Los adultos cuya edad fluctúa entre los 75 84 años fueron quienes refirieron necesitar ayuda. Un $80 \%$ no requiere ayuda para moverse dentro de su casa y el otro $20 \%$ dijo contar con ella. Con respecto a si requería ayuda para vestirse, $86 \%$ dijo no necesitarla y $14 \%$ refirió contar con alguien. Un $60 \%$ dijo s necesitar ayuda para ir al doctor y el 36\% mencionó que requiere contar con alguien quien le ayude a tomar sus medicamentos. Sin embargo, fue significativo para el sexo femenino con $\mathrm{p}<0.05$ quienes requieren de ayuda para tomar sus medicamentos.

\section{Bienestar emocional}

En cuanto a bienestar emocional, el $76 \%$ de adultos mayores considera que toma decisiones importantes que tiene control en su vida. Mientras que el $64 \%$ considera sentirse seguro, el 20\% participa en actividades con otros adultos mayores y sólo el $2 \%$ considera que se siente solo.

\section{Participación social}

Los adultos mayores juegan un papel importante en su red social primaria colaborando en casa con actividades como cocinar o cuidar niños. En cuanto a participar en reuniones con otros adultos mayores, $70 \%$ considera que es muy importante reunirse con otros adultos y $62 \%$ considera muy importante contar con un espacio para hacerlo. Para las mujeres fue significativo reunirse con otros adultos mayores $\mathrm{p}<.05$

En lo que se refiere a participar en una red social secundaria, el $32 \%$ dijo que nunca había participado en grupos de adultos mayores y el $24 \%$ algunas veces. Sin embargo, el $68 \%$ considera importante reunirse con otros adultos mayores y tener espacio para bailar, hace ejercicio, platicar, realizar manualidades, mecánica, carpintería, aprender algo, pintura, corte, jugar domino tomar clases de historia, y poesía.

En estos resultados se puede observar que el adulto mayor en SLA cuenta con redes sociales primarias en donde da y recibe apoyo, lo cual contribuye a su sentido de pertenencia y autoestima. Asimismo, se observa que a pesar del estado de vulnerabilidad en que se encuen tra por el entorno adverso ya descrito y por la etapa de vida que está viviendo, sigue siendo independiente manteniendo una vida productiva y colaborando con el entretejido social.

Los resultados de esta encuesta junto con las aportaciones del grupo parroquial que visita a las personas enfermas como parte de su labor pastoral, constituyeron la base para la elaboración de la Fase II del proyecto.

FASE II: Sensibilización, capacitación y atención a problemas rehabilitatorios que impactan el desempeño ocupacional.

La Fase II tuvo como objetivo general desarrollar un programa para sensibilizar a la comunidad sobre el cuidado de la salud y capacitar a cuidadores primarios en el cuidado de salud del adulto mayor. Sin embargo, la Fase II se amplió para dar atención mediante rehabilitación basada en la comunidad a los adultos que la propia comunidad refiriera, ya que no cuentan con este servicio.

Esta fase llevó una metodología de Investigación Acción - Participación que fue guiada por los siguientes supuestos: la salud concebida como un estado de bienestar físico, mental y social, no sólo como la ausencia de enfermedad (OMS, 1986). El cuidado de la salud se planteó no como una atención "reparativa" y centrada en la enfermedad, sino como una forma de avanzar en el mantenimiento de la salud y el logro de patrones de vida "saludables". Desde la perspectiva ocupacional, se concibió a la salud como "el repertorio de habilidades que e permiten a las gentes alcanzar sus objetivos valorados en sus propios entornos" (Yerxa, 1997).

Se consideró que la participación y la responsabilidad individual y social es uno de los ejes desde donde se produce el cambio en el actuar. Los habitantes de la comunidad y los familiares de los adultos mayores, son los actores principales para el desarrollo de redes de apoyo para el cuidado de la salud, y la conexión e integración de éstos con su comunidad coadyuvaría a mantener su salud y prevenir su deterioro.

Entre las estrategias que se fueron construyendo para cuidar la salud con la comunidad a través de un enfoque de acción/participación se pueden mencionar:

1. Crear, implementar y evaluar un programa de capacitación orientado al cuidador para el cuidado de la salud del adulto mayor. 
2. Desarrollar boletines del cuidado a la salud para apoyar la sensibilización de la población sobre formas de cuidar a los adultos mayores en la propia comunidad, a fin de generar cambios en el ambiente y en la persona.

3. Implementar un programa piloto de atención al adulto mayor con enfermedades crónicas con un enfoque de rehabilitación basada en la comunidad.

Los resultados se evaluaron mediante la metodología de Investigación-Acción a través de la participación activa de los participantes en los proyectos, los testimoniales del adulto mayor y familiares que recibieron atención en RBC, el índice de satisfacción del curso de cuidadores primarios y la aplicación de medidas de seguridad recomendadas en los boletines.

\section{Resultados de la Fase II:}

Los resultados del grupo focal y la entrevista con el Párroco en la Fase I sirvieron de base para modelar el enfoque de la campaña de sensibilización de los boletines y orientar los temas que se incluyeron en los talleres de capacitación de los cuidadores. Ya una vez iniciada la capacitación, las aportaciones de los participantes orientó el contenido de los boletines hacia identificar áreas de riesgo, hacer las casas más seguras y la importancia que tiene el hecho de mantener sus actividades significativas como una forma de cuidar su salud.

\section{La capacitación}

A diferencia de lo que esperábamos, al curso de capacitación no se anotaron adultos mayores, sino cuidadores primarios que habían tenido o tenían la experiencia de trabajar como tales. El taller se enriqueció con la relatoría de los participantes de sus propias experiencias como cuidadores primarios de abuelos, padres, suegros y permitió orientarlo para tratar temas que desconocían como abordar como manejo del duelo, qué hacer cuando al adulto mayor no quiere cooperar para levantarse, tomar sus medicinas o bañarse y disminuir los riesgos de caída cuando se empiezan a manifestar signos de demencia. Conforme avanzó la capacitación, ellos mismos empezaron a referir a los adultos mayores de la comunidad que necesitaban ayuda. Esto nos permitió desarrollar estrategias alternativas para acercarnos a los adultos ma- yores a través de diferentes medios, además de motivar a familiares, cuidadores y voluntarios a participar en las diferentes actividades que se organizaron.

Varios temas de capacitación surgieron de la propia encuesta. En las sesiones se presentaban puntos claves a tratar y conjuntamente con el grupo de capacitación se iba construyendo el conocimiento además de afrontar los retos que se fueron presentando. Uno de ellos fue el que el grupo dejó de contar con el espacio que se nos asignó en principio, pero gracias al apoyo de los participantes, se pudo reinstalar en la propia Oficina Parroquial.

En cuanto a la metodología se utilizó una de carácter participativo para la producción conjunta de conocimiento. Las fases de la misma fueron:1. Introducción, que incluía la presentación de los objetivos de la sesión. 2. Recolección de conceptos, experiencias de las y los participantes en torno al tema de revisión. 3. Ampliación de la perspectiva desde el punto de vista de salud y ocupación. 4. Discusión e integración de aprendizajes.

A fin de conocer el índice de satisfacción de los asistentes al programa de capacitación, se realizó una evaluación al final del proyecto.

Respecto de las actividades realizadas en el proyecto, el $88 \%$ consideró que las de sensibilización desarrolladas a través de boletines informativos y visitas de atención a adultos mayores fueron muy importantes, el $89 \%$ se refirió a las de capacitación en el mismo sentido.

En relación con los temas presentados, el 78\% consideró que les dio herramientas para cuidar mejor al adulto mayor, mantener su movilidad y flexibilidad y resolver los problemas relacionados con la atención que requieren tanto el adulto mayor sano y enfermo, mientras que el $67 \%$ consideró que los temas les dieron herramientas para manejar conflictos y cuidar de su salud.

En cuanto a la necesidad de crear redes de apoyo comunitarias para los adultos mayores, el 89\% consideró necesario continuar con la capacitación para el cuidado de la salud y dar continuidad al Grupo Cuidado de la Salud del Adulto Mayor que se formó durante la capacitación. El 78\% consideró importante organizar una red de alerta para el adulto mayor enfermo y contar con un espacio comunitario.

\section{Visitas domiciliarias de atención a problemas rehabilitatorios.}

Se atendieron un total de 15 personas enfermas, 13 de ellas adultos mayores que presentaban diabetes (3), fractura de cadera (1), osteoartrosis (4), artritis (4), 
problemas respiratorios, hipertensión arterial (4), cirugía intestinal, evento vascular cerebral, hipoacusia, falla cardiaca por válvula mitral, esclerosis múltiple, parálisis cerebral, pérdida de memoria. Se realizaron un promedio de 3 visitas por persona. Se solicitaba que estuviera la familia para educar a los integrantes sobre el problema de salud y destacar la importancia de la red familiar en cuidado de la salud de un adulto mayor.

El tratamiento se orientó a organizar rutinas en su vida diaria, entrenamiento de actividades de vida diaria, higiene postural y de articulaciones, seguridad en el hogar, reducción de riesgos en casa, cómo enseña rutinas de ejercicios para mantener movilidad, técnicas de respiración, involucrar a la familia, mantener su ro del adulto mayor como parte importante en la familia adaptación de sus utensilios y sus herramientas, mantenimiento de roles, uso adecuado del bastón y silla de ruedas.

Los principales resultados que se lograron fueron: retomar ocupaciones que habían dejado de realizar como salir con la familia, integrarse a las actividades parroquiales, participación en la comunidad, disminuir la fatiga y hacer un mejor manejo de sus roles personales. Sólo en un caso se suspendió la visita por un problema cardiaco.

A los dos meses de haber terminado las visitas se visitaron nuevamente a los adultos mayores a quienes se les había dado apoyo con RBC. En esa visita de seguimiento, tanto los familiares como el propio adulto mayor refirieron: "mejoría en su estado de salud". "he hecho las modificaciones a mi casa que nos recomendaron, es más fácil subir las escaleras," "mis rodillas ya no están rígidas, las puedo mover más", "mi calidad de vida mejoró mucho y cambió mi perspectiva sobre el cuidado de la salud", "mejoró su estado de ánimo, ya está caminando más". "ya me siento más tranquilo, ya no podía dormir, ha disminuido la ansiedad", "los ejercicios me han ayudado a moverme mejor" ,"ojalá que puedan continuar las visitas".

\section{Técnicas de análisis}

En el índice de satisfacción se comprobó que el taller les dio herramientas para cuidar la salud de sus familiares, a través de sentirse más competentes en su rol de cuidadores, comprender sus propias necesidades, reconocer el impacto que tiene el que el adulto mayor mantenga el control de su vida a través de decisione ocupacionales. En cuanto a la atención rehabilitatoria los adultos mayores atendidos reportaron haber mejorado en su nivel de involucramiento en ocupaciones de interés, lo cual habla de la necesidad de orientarlos para que, a pesar de padecer enfermedades crónicas, cuenten con orientación para que mantengan sus ocupaciones significativas como una forma de cuidar su salud.

A pesar de que no se contó con un resultado cualitativo del impacto de los boletines en el cuidado de la salud del adulto mayor en la comunidad de Acopilco, el interés con el que leían el boletín mientras esperaban con su familiar el pago de ayuda alimentaria, y los cambios que se observaron en las casas como instalación de barandales y quitar tapetes, indica que la información generó cambios y que se requiere en el futuro estudiar más a fondo estas recomendaciones.

FASE III: Identificación de redes de apoyo para el cuidado a la salud del adulto mayor existentes en la comunidad y tipo de redes que aún se requieren.

El objetivo de la Fase III fue identificar qué redes de apoyo tienen los adultos mayores de para cuidar su salud y que tipos de redes aún se requieren. Para la integración de esta fase se utilizaron los datos obtenidos en la encuesta, el taller de capacitación y también se implementó una metodología de investigación-acción-participación.

\section{Técnicas de análisis}

En el análisis de la encuesta con los participantes de taller mediante grupo focal, sobresalieron varios puntos: En cuanto al adulto mayor., él sigue siendo parte importante del tejido comunitario, porque todavía en buena parte de las familias forma parte de la familia extendida. En este sentido es frecuente que su familia viva en el terrero del abuelo, por lo que es fácil estar cerca de él y estar al pendiente de sus necesidades físicas.

En cuanto a las redes de apoyo, el adulto mayor cuenta con una red primaria de atención y apoyo más desde el punto de vida físico que emocional, que es en donde se empiezan a encontrar casos de abuso por lo que toca a uso de su dinero que recibe del gobierno. El grupo identificó como red secundaria la establecida por SEDESOL, que está disponible para adultos mayores "jóvenes" que se pueden trasladar y tienen interés en tomar las actividades que se ofrecen una vez por semana. En cuanto a su red terciaria reconoció como importante desarrollar un directorio que integre la información de los servicios 
que presta el gobierno a los adultos mayores como un recurso para poder utilizarlos en caso de necesidad.

En cuanto a las áreas de la salud no atendidas en la comunidad, a través de la reflexión de las experiencias vividas, tanto a nivel individual como comunitario, resaltó el hecho que si bien durante el proyecto se habían atendido las necesidades de adultos mayores con necesidades de rehabilitación, esta parte no está siendo cubierta por ninguna de las redes existentes y si bien se logró dar atención durante el tiempo que duró el proyecto, hace falta continuar prestando este tipo de atención en la comunidad.

Asimismo, los asistentes al taller mostraron preocupación por los adultos mayores que están solos y no cuentan redes de apoyo primarias, los cuales aún cuando reciben ayuda del gobierno en cuanto a visitarlos cada dos meses, es insuficiente.

\section{Resultados}

Como producto de esta fase se creó un directorio comunitario en el que se integraron las diferentes instituciones de gobierno que dan atención al adulto mayor (Red Institucional). En él se incluyeron el nombre de la institución, su objetivo, dirección, tipo de apoyo y requisitos para recibirlo. Este directorio fue conformado por todos los participantes del taller de capacitación.

Otro producto fue el inicio de la organización del grupo autogestivo Cuidado de la Salud del Adulto Mayor cuyo principal objetivo será dar continuidad al proyecto, pero ya como un proyecto de la propia comunidad.

En cuanto a la experiencia de aprendizaje en servicio, los estudiantes consideraron que trabajar en la comunidad puso a prueba sus habilidades como terapeutas ocupacionales, ya que, conforme lo expresaron: "requirió abordar el problema de salud desde el punto social, humanitario, involucrándose en la comunidad para comprender las necesidades de salud de sus habitantes."

En relación con lo que aprendieron de esta experiencia, los alumnos mencionaron"la importancia de la participación de los adultos para mantenerse activos, crear redes de apoyo entre las ya existentes y las que pueden formarse a partir del proyecto", "la experiencia que adquirí con cada adulto visitado y cada cuidador me hizo comprender su compromiso con su localidad y sobre todo el esfuerzo individual para mejorar su calidad de vida", "aprendí que se requieren los servicios del terapeuta ocupacional para trabajar con los cuidadores primarios de los adultos mayores en la propia comunidad para mejorar su calidad de vida en lo familiar y en lo social."

\section{DISCUSIÓN}

De acuerdo a Sandoval (2010), los adultos mayores que viven en zonas urbanas marginadas y rurales representan un grupo de población vulnerable, por sus patrones de consumo y estilo de vida que los colocan en grupos de alto riesgo para enfermedades crónico-degenerativas. En este estudio se constató que el adulto mayor de SLA, si bien presenta indicadores de vulnerabilidad por la presencia de enfermedades crónico- degenerativas y el ambiente físico que le hace difícil trasladarse, sigue siendo parte integral de las familias extendidas, en donde se siente respetado y seguro. Sin embargo, ya se empieza a observar que para el cuidador primario es motivo de preocupación no contar con los recursos para atenderlo bien, ya sea por problemas de tiempo, por atender a otras responsabilidades familiares o por la falta de conocimiento acerca del cuidado que requiere el adulto mayor.

Por otra parte, en este estudio se corrobora que el aumento de la esperanza de vida no se correlaciona con mejores condiciones en la calidad de vida, sino con enfermedades o con padecimientos diversos (CEPAL-Celade, 2009, p. 122), lo cual los coloca en una situación vulnerable por el aumento de su dependencia y discapacidad. Los problemas de salud encontrados en esta comunidad, van de acuerdo con lo reportado por (Sánchez Galicia, (2013) y el Instituto Mexicano de Derechos Humanos y Democracia, (2008, p. 107), en cuanto a que $45.9 \%$ viven con alguna discapacidad, contrario a la media $(4.03 \%)$.

En este rubro fue donde más carencias encontramos, ya que la comunidad sólo cuenta con servicios básicos de salud para consultas médicas y no se tienen servicios rehabilitatorios, ni para los adultos mayores, ni para las personas con discapacidad de la comunidad.

El involucramiento activo en ocupaciones con significado y su relación con salud, fue el énfasis de este proyecto que logró impactar al adulto mayor sano, al enfermo, a los cuidadores y a la comunidad en general. Asimismo, fue evidente que para el adulto mayor que empieza a tener problemas de movilidad y deja de asistir y participar, se encuentra en mayor riesgo por lo que se requiere de orientación y servicios especializados para continuar cuidando su salud y realizando ocupaciones significativas. De acuerdo a Ory \& DeFriese (1998) se requiere un reclutamiento activo y "agresivo" para que llegue a los adultos mayores que realmente requieran apoyo, no solamente aquellos que sí pueden asistir a los centros de día. 
En esta investigación se comprobó que el difundir información de salud de una manera sencilla y accesible permite llegar a diferentes sectores de la comunidad y empezar a generar cambios en el cuidado de la salud. El empoderar a los cuidadores reconociendo su conoci miento tácito, tanto de ellos como de los propios adultos mayores, genera mayor participación y contribuye a crea comunidades sanas.

Aun cuando ya hay un programa del gobierno para visitarlos en sus casas, la poca frecuencia de las visitas, el alto número de adultos mayores que se tienen que visita y el énfasis en la enfermedad, ocasiona que este servicio no se traduzca en acciones concretas orientadas a cuida yantener la salud y calidad de vida del adulto mayor. Se requiere implementar sistemas de apoyo efectivos de acuerdo a las necesidades de cada adulto mayor, dar apoyo a la comunidad para que ellos mismos se constituyan en intermediarios de las necesidades de los adultos mayores y los apoyos institucionales que se requieran.

Como bien lo señala SEDESOL (2010) a fin de atende las necesidades de este grupo poblacional, se requieren estrategias inter - institucionales que vinculen a las diferentes dependencias del Gobierno Federal y la distintas Organizaciones No Gubernamentales (ONG) para realizar un trabajo de inclusión social, atender los derechos y mejorar las condiciones de vida de este grupo poblacional.

Esta experiencia vinculó a diferentes actores sociales, tales como adultos mayores, cuidadores primarios, grupos parroquiales, estudiantes de licenciatura y maestría, los cuales contribuyeron con su conocimiento y experiencia a hacer de este proyecto un generador de cambios individuales y grupales que se reflejaron en la toma de conciencia de valorar tanto su propio conocimiento experiencia como la aportación del otro. Todo esto contribuyó a fortalecer el entretejido social de la comunidad. E hecho de vincular la educación superior, la comunidad, el gobierno y a las ONGs es una forma de construir redes de apoyo para el cuidado de la salud del adulto mayor.

Como producto de este proyecto se integró un grupo autogestivo de cuidadores que va a requerir involucrar al adulto mayor y cuidar de no caer en el asistencialismo lo que generaría dependencia y pasividad. Se debe tene presente que las personas deben cuidar de un modo activo su salud y no ser meros receptores de servicios prestados por sistemas institucionales.

Es importante fortalecer el entretejido social para que el adulto mayor siga contando con redes sociales de apoyo tanto primarias, secundarias y terciarias, a fin de que se sienta integrado a la comunidad y siga man- teniendo su salud a través de su propio involucramiento en ocupaciones saludables.

Así mismo, dar atención a los adultos mayores con problemas crónicos que requieren rehabilitación es una prioridad en este tipo de comunidades. Para ello se requiere reiniciar el apoyo de rehabilitación basada en la comunidad para las personas enfermas que necesiten servicios de rehabilitación y no cuenten con ello.

Es necesario continuar difundiendo información sobre cuidados de la salud a toda la comunidad de manera sencilla y accesible. Este estudio, corrobora que la información en salud debe llegar a diferentes sectores de la comunidad para generar cambios en el cuidado de la salud que generen ambientes más seguros para toda la comunidad.

\section{Implicaciones clínicas para terapia ocupacional}

Este tipo de proyectos permitió vincular educación superior, comunidad, gobierno y organizaciones no gubernamentales, lo que genera nuevos campos de acción profesional y desarrollo de competencias profesionales en la comunidad. Asimismo, permitió contar con evidencias acerca de la aportación del terapeuta ocupacional en la construcción de comunidades sanas desde un enfoque de promoción de la salud, y una perspectiva ocupacional que toma en cuenta el involucramiento en ocupaciones y promueve ambientes saludable con los recursos de la comunidad, tomó en cuenta recursos y limitaciones del adulto mayor. Se lograron identificar las prioridades que se deben atender y porqué.

Investigación participativa basada en la comunidad (IPBC) es un enfoque de colaboración que está diseñada para asegurar la participación de las comunidades interesadas en encontrar soluciones a problemas específicos en forma conjunta con instituciones académicas y representantes de organizaciones para mejorar la salud y el bienestar a través de la adopción de medidas, incluyendo el cambio social.

De acuerdo a Viswanathan, 2004, la IPVC implica "Co-aprendizaje y la transferencia recíproca de conocimientos, por todos los actores de la investigación, decisiones compartidas y propiedad mutua de los procesos y productos de la investigación.

Por otra parte, se sentaron las bases para la toma de conciencia de la responsabilidad de continuar trabajando en la comunidad desde la perspectiva ocupacional. 


\section{AgradeCIMIENTOS}

Agradecemos a todas las personas que colaboraron en este proyecto de manera especial al Padre Clemente Santana, a los pasantes de licenciatura Lucero Luciano Castillo y Elizabeth Chávez, por su involucramiento en la atención de los adultos mayores que requerían apoyo rehabilitatorio y en la capacitación y a la Fundación Sertull por su financiamiento para realizar el proyecto en la comunidad.

\section{ReFERENCIAS BibLIOGRÁfICAS}

Antonucci, T. \& Jackson J. (1987). Social support, interpersonal efficacy and health: A life perpective. En Carstense. L. \& Edelstein, B. (Eds), Handbook of clinical gerentology (p. 291-311). New York: Pergamon.

Heaney, C. \& Israel, B. (2008). Social Networks and Social Support. En Glanz, K (Ed). Health Behavior and Health Education: Theory, Research and Practice (p.189-207). San Francisco, CA.

Huenchuan, S. (2009). Envejecimiento, derechos humanos y políticas públicas. Santiago de Chile: Editorial CEPAL.

Huenchuan, S. (2015). Necesidades de Cuidado de las Personas Mayores en la Ciudad de México: diagnostico y lineamientos de política. Recuperado de: http://repositorio.cepal.org/bitstream/handle/11362/38879/ S1500754_es.pdf?sequence $=1 \&$ isAllowed $=y$

Clark, F. (1997). Occupational therapy for independent-living older adults: A randomized controlled trial. Journal of the American Medical Association, (278) 1321-1326. Doi:10.1001/jama.1997.03550160041036.

Cummings, R. (1999). Home visits by an occupational therapist for assessment and modification of environmental hazards: a randomized trial of falls prevention. Journal American Geriatric Society. 47(12), 1397- 1402.

CONAPO. (2015). Proyecciones de la Población de México, 2000-2050. Recuperado de http://www.conapo.gob.mx/es/CONAPO/Proyecciones

Doll, J. (2010). Community Practice in Occupational Therapy: What is $i t$ ?. En, J. Doll (1 ${ }^{\mathrm{st}}$, Ed). Program Development and Grant Writing in Occupational Therapy: Making the connection. (pp. 1- 23). Ontario Canada: Jones \& Bartlett Learning.

GutiérrezJ.P. Rivera-Dommarco J, Shamah-Levy T., Villalpando-Hernández S., Franco A., Cuevas-Nasu L, Romero-Martínez M, Hernández-Ávila M. (2012). Encuesta Nacional de Salud y Nutrición. Recuperado de: http://ensanut.insp.mx/doctos/analiticos/DiscapacidAdultMayor.pd

Fazio, L. (2nd ed.). (2008). Developing occupation-centered programs for the community. Upper Saddle River, NJ: Prentice Hall Health.

Fidler, G. (2001). Community Pratice: It's more tan geography. Occupationa/ Therapy in Health Care. 13 (3/4), 7-9. Doi.org/10.1080/J003v13n03 02

Fundación Cáritas para el Bienestar del Adulto Mayor I.A.P. (2005). Redes de las Redes Sociales de Apoyo de los Adultos Mayores: Guía de Aprendizaje No Formal para Adultos Mayores. Recuperado de: http://www.gerontologia.org/portal/archivosUpload/Guia_Redes_Adulto_Mayor.pdf
Gitlin, L.N. (2009). Environmental Adaptations for Older Adults and Their Families in the home and Community. En Söderback (Ed.1 $1^{\mathrm{ST}}$ ). International Handbook of Occupational Therapy Interventions (pp.53-62). London, United Kingdon: Springer.

Glanz, K., Rimer. B.K. \& Viswanath, K. (4 ${ }^{\text {th }}$ Ed) (2008). Health Behavior and Health Education: Theory, San Francisco California: Josey-Bass.

Graff, M. (2006). Community based occupational therapy for patients with dementia and their care givers: randomized controlled trial. British Medical Journal. (7580), 1196

Graff, M. (2008). Community occupational therapy for older patients with dementia and their caregivers: cost effectiveness study. British Medical Journal. 336(7636), 134-8. Doi: 10.1136/bmj.39408.481898.BE Guzman, J.M., Huenchuan, S. y Montes de Oca, V. (Julio, 2003). Redes de Apoyo Social de las Personas Mayores: Marco Conceptual. Viejos y Viejas. Participación, Ciudadanía e Inclusión Social. 51 Congreso Internacional de Americanista. CEPAL, Santiago de Chile

INEGI. (2014). Encuesta Nacional de Ingresos y Gastos de los Hogares 2014. Recuperado: http://internet.contenidos.inegi.org.mx/contenidos/productos//prod_serv/contenidos/espanol/bvinegi/productos/ metodologias/ENIGH/ENIGH2012/702825051075.pdf

INEGI. (2013). Estadísticas a Propósito del Día Internacional de las Personas con Discapacidad. Recuperado de https://www.gob.mx/cms/ uploads/attachment/file/9640/inegi_2012.pdf

INEGI. (2013). Encuesta Nacional de Empleo y Seguridad Social 2013. ENESS. Recuperado de http://internet.contenidos.inegi.org.mx/contenidos/productos//prod_serv/contenidos/espanol/bvinegi/productos/ encuestas/hogares/eness/2014/702825058777.pdf

Montes, B. (2008). Envejecimiento de la población en México: perspectivas y retos desde los Derechos Humanos. Recuperado de http://www. imdhd.org/doctos/EnvejecimientoMexico-1.pdf.

Issac, D. (1990). Community Occupational Therapy with Mentally Handicapped Adults. London England. Edit. Chapman and Hall.

Letts, L. (2011). Promoción de la salud. En E. Crepeau (11 Ed.), Willard and Spackman: Terapia ocupacional (pp 165-180). Buenos Aires, Argentina: Editorial Panamericana.

Liddle, J. (1996). Can occupational therapy intervention play a part in maintaining independence and quality of life in older people? A randomised controlled trial. Australian and New Zealand Journal of Public Health. 20(6), 574-578. Doi:10.1111/j.1467-842X.1996.tb01068.x

McColl, M. (1998). What do you need to know to practice occupational therapy in community? American Journal of Occupational Therapy. (52) 60-64. Doi: 10.5014/ajot.52.1.11

Matuska, K. (2003). Outcomes of a Pilot Occupational Therapy Wellness Program for Older Adults. The American Journal of Occupational Therapy, (52) 220-224 Doi: 10.5014/ajot.57.2.220

Ory, M.G. \& DeFriese, G.H. (1998). Self-Care in Later Life. New York: Springer Publishing Co.

Khasnabis Ch. (2014). Rehabilitación Basada en la Comunidad. Recuperado de www.who.int/disabilities/cbr/es/Organización Mundial de la Salud. (1986). Carta de Ottawa para la Promoción de la Salud. 
Ottawa, 21 November. Recuperado de http://www1.paho.org/ spanish/HPP/OttawaCharterSp.pdf

Sanchez, C (2013). Investigación-AcciónParticipación en una Comunidad Rural desde la Perspectiva Ocupacional en Gerontología. Tesis para obtener el grado de maestria en Terapia Ocupacional. Instituto de Terapia Ocupacional, Ciudad de México

Sandoval, H. (Mayo, 2010). Impacto de las enfermedades crónicas en las poblaciones pensionadas. En Seminario de Pensiones 2010. Seminario dirijido por Instituto Nacional de Salud Pública 2010, México DF.

SEDESOL. (2010). Diagnóstico sobre la situación de vulnerabilidad de la población de 70 años y más. Recuperado en: http://www.sedesol. gob.mx/work/models/SEDESOL/Sedesol/sppe/dgap/diagnostico/ Diagnostico_70\%20y\%20Mas_VERSION_FINAL.pdf

Scaffa, M. (2001). Occupational Therapy in Community Based Practice Settings. Philadelphia: Davies Co. Prentice Hall.

Viswanathan, M., Ammerman, A., Eng, E. (2004). Community-Based Participatory Research: Assessing the Evidence. Evidence report Technology, 8(99), 1-8.

Yerxa, E. (1998). Occupation: The Keystone of a Curriculum for a Self-Defined Profession. The American Journal of Occupational Therapy, 52(5), 365- 372. Doi:10.5014/ajot.52.5.365 